\title{
Subtractive 3D Printing of Optically Active Diamond Structures
}

SUBJECT AREAS: MATERIALS FOR OPTICS MATERIALS FOR DEVICES

Received

28 January 2014

Accepted

2 May 2014

Published

21 May 2014

Correspondence and requests for materials should be addressed to M.T. (milos.toth@uts. edu.au) or I.A. (igor. aharonovich@uts.edu.

\author{
Aiden A. Martin, Milos Toth \& Igor Aharonovich
}

School of Physics and Advanced Materials, University of Technology, Sydney, P.O. Box 123, Broadway, New South Wales 2007, Australia.

Controlled fabrication of semiconductor nanostructures is an essential step in engineering of high performance photonic and optoelectronic devices. Diamond in particular has recently attracted considerable attention as a promising platform for quantum technologies, photonics and high resolution sensing applications. Here we demonstrate the fabrication of optically active, functional diamond structures using gas-mediated electron beam induced etching (EBIE). The technique achieves dry chemical etching at room temperature through the dissociation of surface-adsorbed $\mathrm{H}_{2} \mathrm{O}$ molecules by energetic electrons in a water vapor environment. Parallel processing is possible by electron flood exposure and the use of an etch mask, while high resolution, mask-free, iterative editing is demonstrated by direct write etching of inclined facets of diamond microparticles. The realized structures demonstrate the potential of EBIE for the fabrication of optically active structures in diamond.

D

iamond, long considered unconquerable due to its extraordinary strength and chemical resistance, has found applications across numerous areas of science due to its unique combination of optical, electronic, chemical and thermal properties ${ }^{1}$. Most notably, the nitrogen vacancy luminescence center (NV) has been employed as a spin qubit, enabling the use of diamond as a platform for next generation sensing, nanophotonic and quantum devices ${ }^{2-8}$. These tantalizing applications are, however, overshadowed by challenges in fabrication arising from its extraordinary hardness and chemical resistance.

At present, diamond fabrication requires cumbersome masking techniques, and ion bombardment or high power laser ablation, which often causes damage and material redeposition artifacts ${ }^{9-13}$. Fabrication and editing of optoelectronic grade nanostructures is therefore extremely limited relative to conventional semiconductors such as silicon and gallium arsenide. Furthermore, direct-write, deterministic patterning of optical structures in diamond has not been demonstrated in the absence of severe surface damage caused by ion implantation and redeposition of non-volatile, sputtered or ablated material.

Here we demonstrate the fabrication of functional, optically active diamond structures using gas-mediated electron beam induced etching (EBIE) ${ }^{14-18}$ (Figure 1). EBIE achieves dry chemical etching at room temperature in a water vapor environment through the electron-induced dissociation of surface-adsorbed $\mathrm{H}_{2} \mathrm{O}$ molecules, generating reactive fragments that give rise to volatilization of carbon. The reaction steps are shown in Figure $1 \mathrm{~b}-\mathrm{c}$. The process utilizes low energy electrons which do not cause damage through knock-on displacement of carbon, sputtering and staining that are characteristic of focused ion beam milling, and cause quenching of diamond luminescence ${ }^{14,19}$. Parallel processing is possible by electron flood exposure and the use of an etch mask. Mask-free EBIE is used to realize direct-write subtractive 3D printing of diamond nanostructures on inclined planes of diamond microparticles. The processes are demonstrated using a variable pressure scanning electron microscope (SEM) making diamond nanofabrication accessible to most nanotechnology laboratories in the world.

To demonstrate the applicability of EBIE to device fabrication, we start by fabricating a pillar from a single crystal diamond using an etch mask. Pillars are used as antennas that enhance light extraction from embedded emitters, particularly of high refractive index semiconductors. The EBIE process is shown schematically in Figure 2a-c. The mask must either absorb the incident electrons or prevent $\mathrm{H}_{2} \mathrm{O}$ from adsorbing to the diamond substrate. Here we use a silica mask to prevent low-energy $(2 \mathrm{keV})$ electrons from penetrating into underlying regions of diamond. The resulting pillars (Figure 2d) have high aspect ratios and straight side-walls, making them ideal for photonic applications. The minimum pillar diameter is ultimately limited by the diameter of the interaction volume of a delta function electron beam, which scales super-linearly ${ }^{20}$ with electron energy. In diamond, it is equal to $\sim 19 \mathrm{~nm}$ at $2 \mathrm{keV}$, and $\sim 9 \mathrm{~nm}$ at $1 \mathrm{keV}$, as shown in Figure $2 \mathrm{e}^{21}$. Nanostructures can 


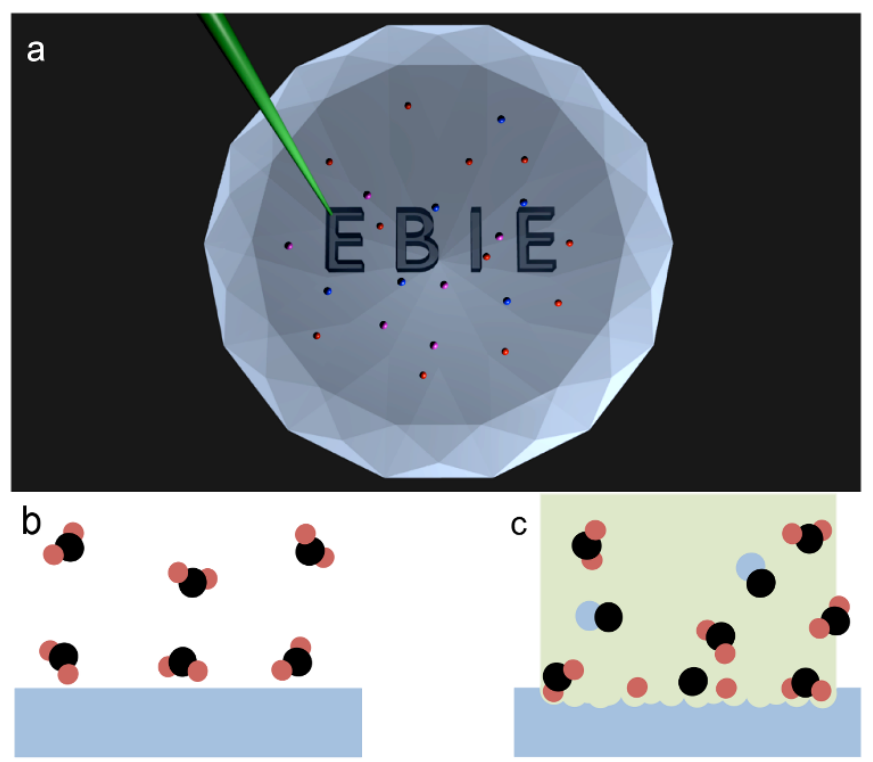

Figure $1 \mid$ Schematic illustrations of $\mathrm{H}_{2} \mathrm{O}$ mediated electron beam induced etching. (a) Direct-write subtractive printing of diamond. (b-c) Volatilization of diamond by electron exposure in a gaseous $\mathrm{H}_{2} \mathrm{O}$ environment.

therefore be fabricated with high resolution using the correct combination of mask diameter and electron energy. On the other hand, micron sized depths can be achieved, enabling high aspect ratio geometries.

Optical properties of the pillars are shown by the fluorescence and Raman scattering data in Figure 3. Photoluminescence (PL) spectra were recorded using a confocal microscope with a $532 \mathrm{~nm}$ excitation laser. The PL intensity of the pillar (Figure 3a) shows a two-fold increase over the neighboring, unprocessed region of diamond (under identical PL collection conditions). Raman spectroscopy (Figure $3 b$ ) shows no evidence of graphitic inclusions in the irradiated area with the first-order diamond peak positioned at $1332 \mathrm{~cm}^{-1}$ and FWHM of $\sim 3.6 \mathrm{~cm}^{-1}$, consistent with the Raman signature of pristine, single crystal diamond ${ }^{22,23}$.

Next, we demonstrate the capability of EBIE for mask-free editing of inclined diamond surfaces. Editing of multiple inclined facets is nearly impossible by mask-based processing techniques, including electron- and photo-lithography. To demonstrate the three dimensional capability of writing on inclined surfaces, we patterned the letters 'UTS' and 'NANO' into individual microparticles (Figure 4) simply by tracing out the letters using an electron beam as shown schematically in Figure 1d. Etching was carried out using a $20 \mathrm{keV}$ electron beam, while charging was stabilized using a low vacuum (13 Pa) environment of $\mathrm{H}_{2} \mathrm{O}$. The letters are clearly visible in SEM images (Figure 4a), while atomic force microscope (AFM) maps of the 'UTS' logo show line widths and depths of $\sim 100 \mathrm{~nm}$ (Figure 4b). The letters 'NANO' were written intentionally across three diamond (111) facets, showing the ability of EBIE to edit three dimensional, inclined nanostructures. Figure $4 \mathrm{c}$ shows an individual diamond microparticle with visible (111) facets and Figure $4 \mathrm{e}$ shows the word 'NANO' imprinted in the crystal, with the letters 'NA', ' $\mathrm{N}$ ' and 'O', occupying all three (111) planes, respectively. PL measurements recorded from the diamond microparticles exhibit strong fluorescence, confirming that the etch process does not destroy optical properties and material functionality (See Supplementary Information). The mask-less patterning approach is particularly attractive for generation of high resolution microfluidic channels in microdiamond crystals, in close proximity to optical emitters ${ }^{24,25}$.
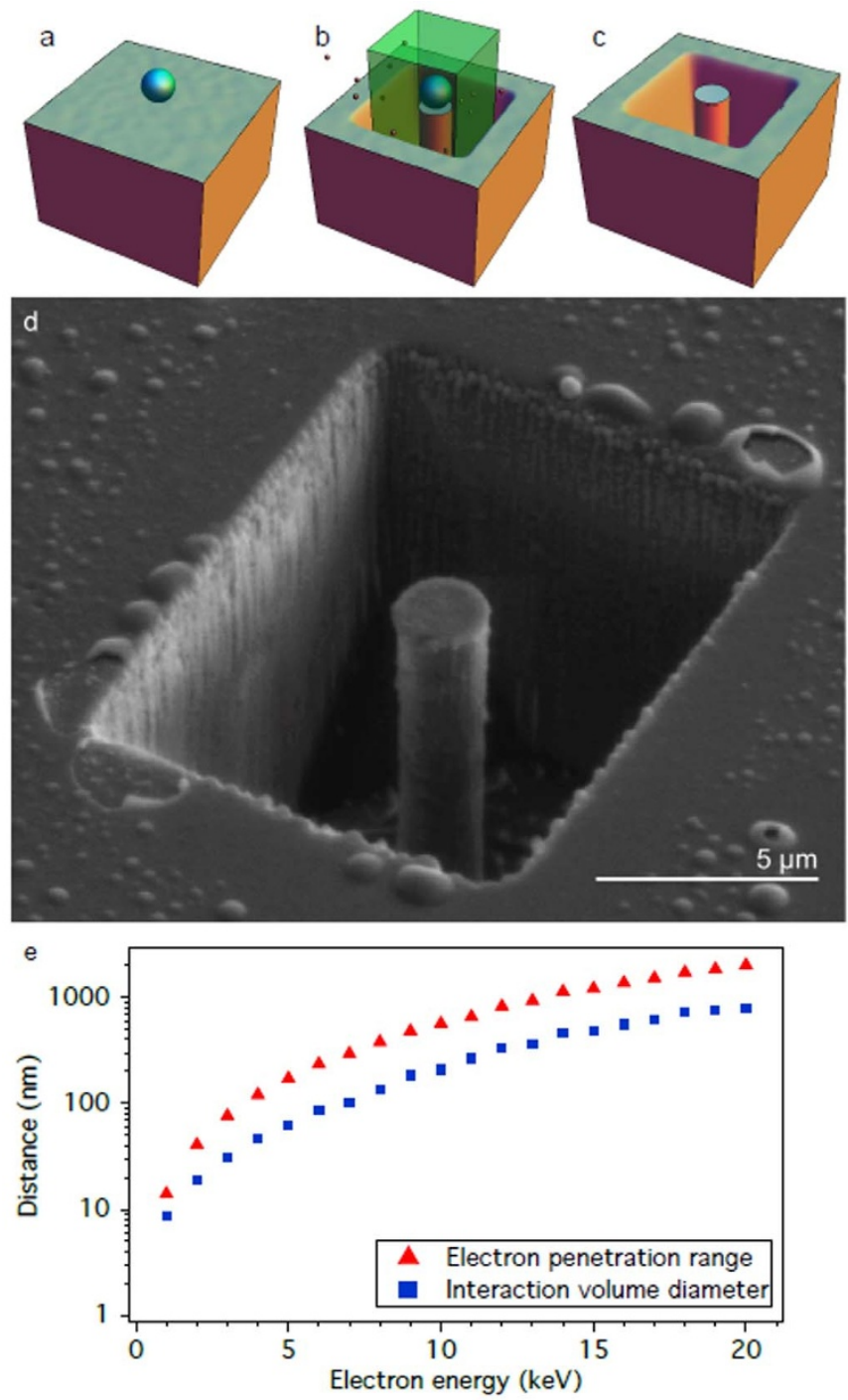

Figure $2 \mid$ Diamond pillar fabricated by mask-based EBIE.

(a-c) Schematic illustration of pillar fabrication by $\mathrm{H}_{2} \mathrm{O}$ mediated EBIE. (a) Silica bead on a diamond surface. (b) Diamond volatilization achieved by scanning a $2 \mathrm{keV}$ electron beam over a rectangle repeatedly in a $\mathrm{H}_{2} \mathrm{O}$ environment. The silica bead acts as an etch mask that prevents the electrons from reaching the diamond surface. (c) Final pillar geometry after the silica bead was removed from the substrate. (d) Electron image of a pillar fabricated in single crystal diamond by $\mathrm{H}_{2} \mathrm{O}$-mediated EBIE using the process shown in a-c. (e) Depth and diameter of the electron interaction volume that contains $90 \%$ of the energy deposited into diamond, plotted as a function of electron energy. The values were calculated using a Monte Carlo model of electron-solid interactions.

The potential of EBIE exceeds that of traditional etching techniques for wide bandgap semiconductors. For instance, a combination of EBIE with cathodoluminescence analysis techniques may enable probing of the spectroscopic properties of nanostructures while the etch parameters are modified during fabrication. Alternatively, substrate tilting can enable fabrication of undercut structures that are currently not available in diamond. Finally, the EBIE method will be pivotal for realizing hybrid devices when direct sculpting of a nanostructure is required to achieve close proximity with an external cavity or metallic nanostructure ${ }^{26}$.

We have demonstrated a promising approach to pattern and sculpt optically active diamond structures using two variants of $\mathrm{H}_{2} \mathrm{O}$-mediated electron induced chemical etching: a mask-based 


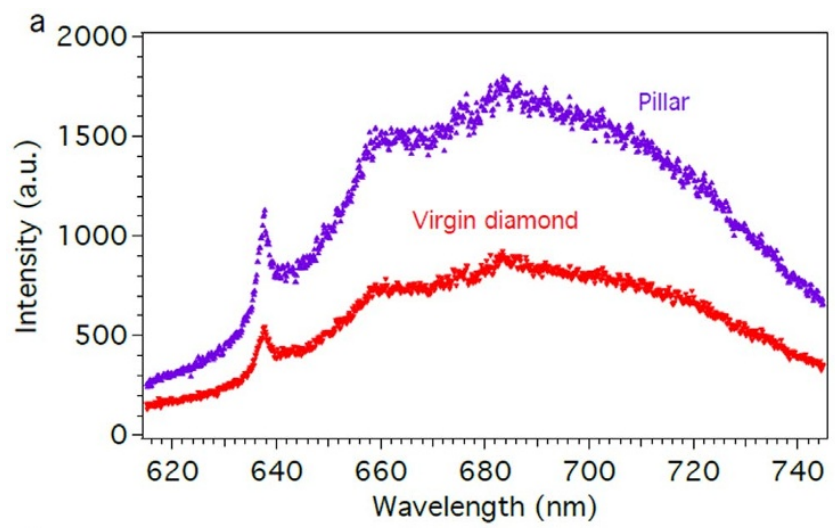

b

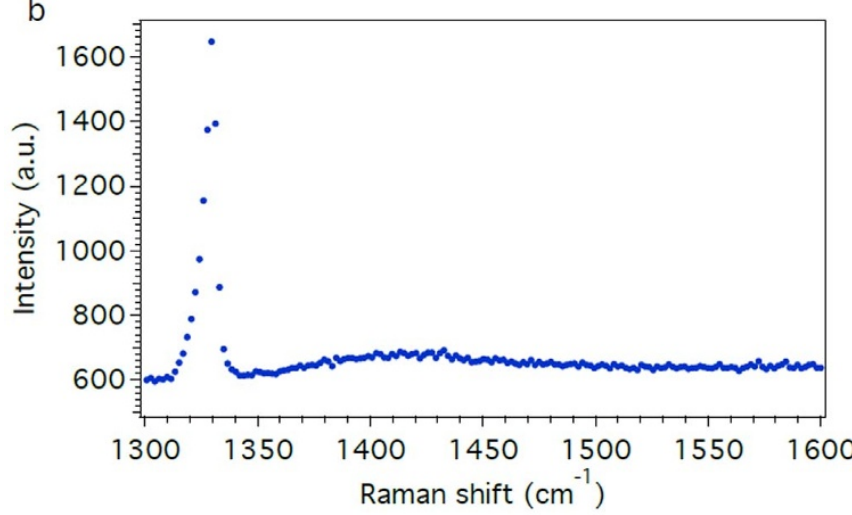

Figure 3 Optical quality of a diamond pillar fabricated by EBIE. (a) PL spectra of the pillar and an adjacent, unprocessed region of diamond. (b) Raman spectrum of the pillar showing the absence of defects generated by EBIE. lithographic approach, and an extremely versatile, direct-write editing process. For the first time, direct $3 \mathrm{D}$ writing is realized on various facets of a single microparticle. PL and Raman analysis were used to show that the unique optical properties of diamond are maintained and no graphitization occurs. By leveraging the advanced functionalities provided by a conventional SEM in conjunction with EBIE a modification to existing devices and direct nanofabrication for rapid prototyping is enabled. EBIE is the first step towards rendering $3 \mathrm{D}$ single crystal diamond geometries for high performance photonic, sensing and quantum devices.

\section{Methods}

EBIE of diamond pillar. EBIE was performed using a FEI Nova NanoSEM variable pressure $^{27}$ scanning electron microscope (SEM) equipped with a magnetic immersion lens gaseous secondary electron detector ${ }^{28}$. The SEM chamber was pumped to $3 \times$ $10^{-4}$ Pa prior to performing EBIE at room temperature under $13 \mathrm{~Pa}^{\circ} \mathrm{H}_{2} \mathrm{O}$ (Milli-Q) precursor vapor. Pillars were fabricated using dispersed $2 \mu \mathrm{m}$ silica beads that served as a hard mask on a 100 oriented single crystal diamond (Element Six). Silica beads were transferred from a suspension in isopropyl alcohol, which was flash dried to remove the solvent. Single beads were located in the SEM before irradiation. $\mathrm{H}_{2} \mathrm{O}$ mediated EBIE was performed by irradiating a $10 \mu \mathrm{m} \times 8 \mu \mathrm{m}$ rectangle with a $2 \mathrm{keV}$, $29.9 \mathrm{nA}$ electron beam for 12 hours, using a dwell time of $1.34 \mathrm{~ms}$ per pixel. Electron penetration and energy deposition profiles were calculated using standard Monte Carlo models of electron-solid interactions ${ }^{21,29}$.

Optical measurements. PL measurements were performed at room temperature using a custom built confocal microscope with a $532 \mathrm{~nm}$ excitation laser. Raman measurements were performed using a Renishaw inVia Raman microscope with a $633 \mathrm{~nm}$ excitation laser.

EBIE of diamond micro particles. Diamond micro-particles were grown using a CVD method ( $\sim 950 \mathrm{~W}, 1 \%$ methane, $\left.8 \times 10^{3} \mathrm{~Pa}\right)$. The particles were transferred to a platinum surface prior to EBIE. The 'UTS' symbol was etched into a single particle using a $20 \mathrm{keV}, 9.9 \mathrm{nA}$ electron beam controlled using a custom pattern generator connected to the SEM scan coils. The dwell time per pixel was $256 \mathrm{~ms}$ and the total process time $\sim 15$ mins. The symbol 'NANO' was etched using the same conditions, but etch letter was etched individually into the diamond. The 'UTS' symbol was measured ex situ using the tapping mode of a DI Dimension 3100 atomic force microscope (AFM), and analyzed using the software package Gwyddion ${ }^{30}$.

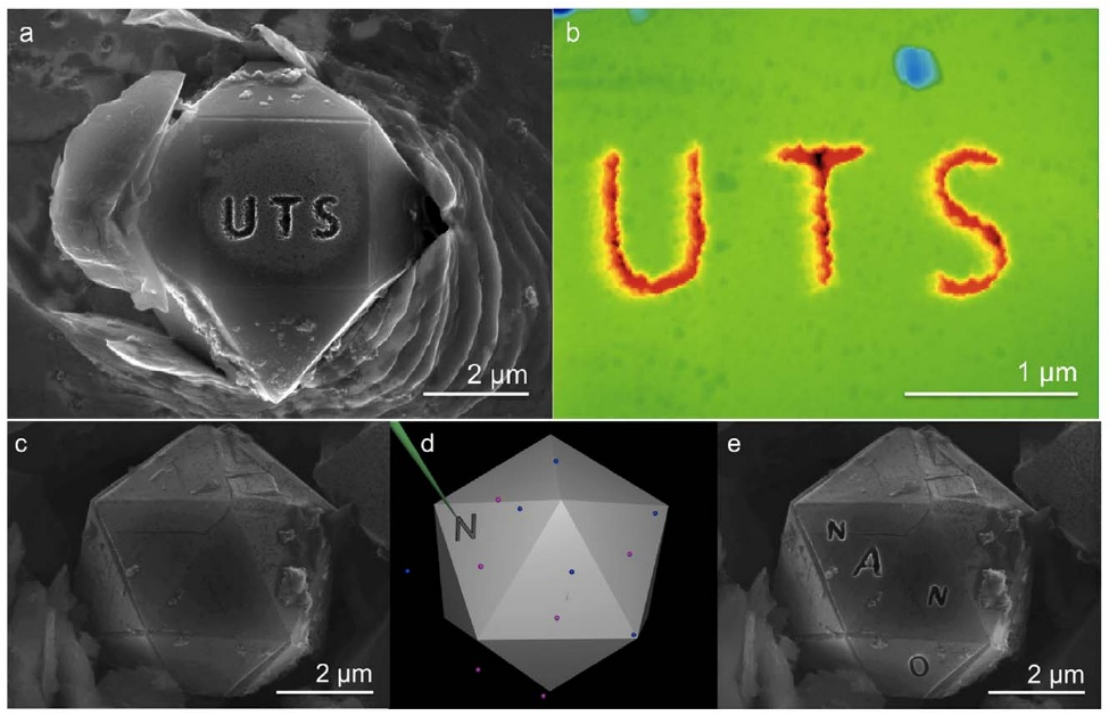

Figure $4 \mid$ Beam-directed editing of Si-doped diamond micro-particles. (a) SEM image of the symbol 'UTS' patterned by $\mathrm{H}_{2} \mathrm{O}$ mediated EBIE on the 110 plane of a single diamond micro-particle embedded in platinum. (b) AFM image of the symbol 'UTS' shown in a (depth of each letter $\sim 100$ nm). (c) SEM image of a diamond micro-particle. (d) Schematic illustration of the process used to pattern the micro-particle shown in c. Each letter of 'NANO' was patterned individually using $\mathrm{H}_{2} \mathrm{O}$ mediated EBIE on three different 111 faces of diamond. (e) SEM image of the microparticle shown in c after the letters 'NANO' were patterned by EBIE. 
1. Wort, C. J. H. \& Balmer, R. S. Diamond as an electronic material. Mater. Today 11, 22-28 (2008).

2. Kaufmann, S. et al. Detection of atomic spin labels in a lipid bilayer using a singlespin nanodiamond probe. Proc. Natl. Acad. Sci. U. S. A. 110, 10894-10898 (2013).

3. Maletinsky, P. et al. A robust scanning diamond sensor for nanoscale imaging with single nitrogen-vacancy centres. Nat. Nanotechnol. 7, 320-324 (2012).

4. Riedrich-Moller, J. et al. One- and two-dimensional photonic crystal microcavities in single crystal diamond. Nat. Nanotechnol. 7, 69-74 (2012).

5. Zhu, X. et al. Coherent coupling of a superconducting flux qubit to an electron spin ensemble in diamond. Nature 478, 221-224 (2011).

6. Dobrovitski, V. V., Fuchs, G. D., Falk, A. L., Santori, C. \& Awschalom, D. D. Quantum Control over Single Spins in Diamond. Annu. Rev. Condens. Matter Phys. 4, 23-50 (2013).

7. Childress, L. \& Hanson, R. Diamond NV centers for quantum computing and quantum networks. MRS Bull. 38, 134-138 (2013).

8. Andrei, F. et al. Quantum photonic devices in single-crystal diamond. New J. Phys. 15, 025010 (2013).

9. Burek, M. J. et al. Free-Standing Mechanical and Photonic Nanostructures in Single-Crystal Diamond. Nano Lett. (2012).

10. Hausmann, B. J. M. et al. Integrated High-Quality Factor Optical Resonators in Diamond. Nano Lett. (2013).

11. Aharonovich, I. et al. Homoepitaxial Growth of Single Crystal Diamond Membranes for Quantum Information Processing. Adv. Mater. 24, OP54-OP59 (2012).

12. Tao, Y. \& Degen, C. Facile Fabrication of Single-Crystal-Diamond Nanostructures with Ultrahigh Aspect Ratio. Adv. Mater. 25, 3962-3967 (2013).

13. Bayn, I., Meyler, B., Salzman, J. \& Kalish, R. Triangular nanobeam photonic cavities in single-crystal diamond. New J. Phys. 13, 025018 (2011).

14. Utke, I., Moshkalev, S. \& Russell, P. Nanofabrication Using Focused Ion and Electron Beams. Oxford University Press, New York, New York (2012).

15. Randolph, S. J., Fowlkes, J. D. \& Rack, P. D. Focused, Nanoscale Electron-BeamInduced Deposition and Etching. Crit. Rev. Solid State Mater. Sci. 31, 55-89 (2006).

16. Martin, A. A., Phillips, M. R. \& Toth, M. Dynamic Surface Site Activation: A Rate Limiting Process in Electron Beam Induced Etching. ACS Appl. Mater. Interfaces 5, 8002-8007 (2013).

17. Taniguchi, J., Miyamoto, I., Ohno, N. \& Honda, S. Electron Beam Assisted Chemical Etching of Single Crystal Diamond Substrates. Jpn. J. Appl. Phys. 35, 6574 (1996).

18. Niitsuma, J., Yuan, X., Koizumi, S. \& Sekiguchi, T. Nanoprocessing of Diamond Using a Variable Pressure Scanning Electron Microscope. Jpn. J. Appl. Phys. 45, L71 (2006).

19. Bayn, I. et al. Diamond processing by focused ion beam - surface damage and recovery. Appl. Phys. Lett. 99, 183109 (2011).

20. Kanaya, K. \& Okayama, S. Penetration and energy-loss theory of electrons in solid targets. J. Phys. D: Appl. Phys. 5, 43 (1972).

21. Hovington, P., Drouin, D. \& Gauvin, R. CASINO: A new monte carlo code in C language for electron beam interaction - part I: Description of the program. Scanning 19, 1-14 (1997).
22. Magyar, A. et al. Fabrication of Thin, Luminescent, Single-crystal Diamond Membranes. Appl. Phys. Lett. 99, 081913 (2011).

23. Orwa, J. O., Nugent, K. W., Jamieson, D. N. \& Prawer, S. Raman investigation of damage caused by deep ion implantation in diamond. Phys. Rev. B: Condens. Matter Mater. Phys. 62, 5461-5472 (2000).

24. Ziem, F. C., Götz, N. S., Zappe, A., Steinert, S. \& Wrachtrup, J. Highly Sensitive Detection of Physiological Spins in a Microfluidic Device. Nano Lett. 13, 4093-4098 (2013).

25. Ohashi, K. et al. Negatively Charged Nitrogen-Vacancy Centers in a $5 \mathrm{~nm}$ Thin 12C Diamond Film. Nano Lett. 13, 4733-4738 (2013).

26. Benson, O. Assembly of hybrid photonic architectures from nanophotonic constituents. Nature 480, 193-199 (2011).

27. Danilatos, G. D. Foundations of environmental scanning electron microscopy. Adv. Electron. Electron Phys. 71, 109-250 (1988).

28. Thiel, B. L. et al. Two-stage gas amplifier for ultrahigh resolution low vacuum scanning electron microscopy. Rev. Sci. Instrum. 77, - (2006).

29. Toth, M. \& Phillips, M. R. Monte Carlo modeling of cathodoluminescence generation using electron energy loss curves. Scanning 20, 425-432 (1998).

30. Nečas, D. \& Klapetek, P. Gwyddion: an open-source software for SPM data analysis. Cent. Eur. J. Phys. 10, 181-188 (2012).

\section{Acknowledgments}

This work was partly funded by FEI Company. A.A.M. is the recipient of a John Stocker Postgraduate Scholarship from the Science and Industry Endowment Fund. I.A. is the recipient of an Australian Research Council Discovery Early Career Research Award (Project Number DE130100592)

\section{Author contributions}

A.M. performed the experiments, and wrote the paper with contributions from all co-authors. A.M., M.T. and I.A. discussed the data and analyzed the results.

\section{Additional information}

Supplementary information accompanies this paper at http://www.nature.com/ scientificreports

Competing financial interests: The authors declare no competing financial interests. How to cite this article: Martin, A.A., Toth, M. \& Aharonovich, I. Subtractive 3D Printing of Optically Active Diamond Structures. Sci. Rep. 4, 5022; DOI:10.1038/srep05022 (2014).

This work is licensed under a Creative Commons Attribution-NonCommercialNoDerivs 3.0 Unported License. The images in this article are included in the article's Creative Commons license, unless indicated otherwise in the image credit; if the image is not included under the Creative Commons license, users will need to obtain permission from the license holder in order to reproduce the image. To view a copy of this license, visit http://creativecommons.org/licenses/by-nc-nd/3.0/ 